and being loved. Based on the high prevalence of love in the lives of older adults and the enduring nature of love over time, our findings support the view that love is a cultural universal. With more and more people living to reach old-age, recognizing the almost universal potential of older adults for loving and being loved become important challenges to disavowing ageism and for maintaining a civil society.

\section{AGEIST STEREOTYPES AND THE INTERGENERATIONAL REENGAGEMENT OF OLDER ADULTS}

G.D. Rowles ${ }^{1}$, M.A. Guest ${ }^{1}$, T. Kruger ${ }^{2}$, A.M. Schuster ${ }^{1}$, K.A. Anderson ${ }^{3}$, K.A. Nikzad-Terhune ${ }^{4}$, V. Lawson ${ }^{1}, 1$. University of Kentucky, Lexington, Kentucky, 2. Indiana State University, Terre Haute, Indiana, 3. University of Montana, Missoula, Montana, 4. Northern Kentucky University, Highland Heights, Kentucky

In many traditional societies across the world, aging is viewed positively and older adults are fully integrated into their community, holding positions of influence and respect. In contrast, gerontophobia and gerontological illiteracy in most developed societies reinforces ageism--prejudice and discrimination against older adults--that estranges generations. In this three year study we examine the perceptions and representations of aging and old age of 1,034 undergraduate students completing introductory courses at six universities across the United States. The study examined views and representations of aging and old age. Students were asked to provide an age at which they considered an individual to be old, to list words they associated with "old person" and "grandma/grandpa," and to draw a sketch representing "aging." Findings indicate that (1) students consider a person to be old in the United States at 65 years of age, (2) have a far more positive view of older adults they know personally (i.e. their grandparents) than older adults as a category; and (3) images of aging, as represented in hand drawn sketches, are predominantly negative; they focus on a relatively short period at the end of life and are pervaded by concern for time, physical decline, medical care, institutionalization, and death. The findings reinforce the need for renewed efforts in developed societies to combat age stereotyping and ageism through gerontological education in order to facilitate the intergenerational reengagement of older adults as lifelong contributors to community life and culture.

\section{RACIAL DIFFERENCES IN ATTITUDES TOWARD AGING, AGING KNOWLEDGE, AND CONTACT R.C. Intrieri, M.L. Kurth, Psychology, Western Illinois University, Macomb, Illinois}

Ageism is defined as "the systematic stereotyping of and discrimination against older people, because they are old, just as racism and sexism have accomplished this with skin color and gender" (Butler, 1975). Ageism in the twenty-first century may be more compelling than ever because of the sheer number of Baby Boomers turning 65 daily. Current population estimates suggest that the percentage of older adults will grow to about $18 \%$ of the population by 2030 (Cohn $\&$ Taylor, 2010). It is believed that misconceptions about aging are developed and maintained due to misinformation about aging and lack of meaningful contact with older adults. Butler's acknowledgment of ageism as a systemic problem has generated research attempting to develop an adequate way to measure ageism, knowledge about aging, and attitudes toward older people. This research examined differences in attitudes toward aging, knowledge of aging and contact frequency between non-Hispanic whites and African-American young adults. Participants were 96 African-American (Mean age 19.27, $S D=1.318$ ) and 175 Non-Hispanic Whites (Mean age 19.87, $S D=1.685$ ) that were part of a larger study. ANOVA revealed significant group differences on the Aging Semantic Differential-Instrumental factor $\left(\mathrm{F}_{(1,269}\right)=8.169$, $\mathrm{p}=.005)$ and on the Fraboni Scale-Anitlocution factor $\left(\mathrm{F}_{11}\right.$ $\left.{ }_{269)}=13.117, \mathrm{p}<.0001\right)$. No significant differences emerged between groups on either the Facts on Aging Quiz-Revised or everyday contact with older adults. Results suggest that while Non-Hispanic Whites held more negative attitudes toward older adults and higher levels of ageism they also showed higher levels of knowledge and a lower frequency of elder contact.

\section{PERSONAL BELIEFS AND REACTIONS TO AGE AND MEMORY THREAT IN EVERYDAY MATERIALS}

C.M. Strickland-Hughes ${ }^{1}$, R.L. West ${ }^{2}$, 1. Psychology, University of Florida, Gainesville, Florida, 2. University of Florida, Gainesville, Florida

Older adults hold complex, yet predominantly negative, beliefs about aging. When experimenters emphasize negative stereotypes about aging, it can be a threat, affecting older adults' physical performance, self-perceptions of aging, and memory. Reactions to stereotyping may vary with the situation or the individual. This study examines the impact of stereotype exposure for the first time with common everyday materials and evaluates the impact of stereotype exposure on personal beliefs and memory. A priori personal beliefs (e.g., sense of control over memory) may promote resilience to stereotypes, and beliefs may change after stereotype exposure. Participants were 34 men and women (ages 50-75; $M=62$ ). Everyday stimuli were newspaper cartoons, "jumble" puzzles, and word searches. A stereotype group saw stimuli with embedded age (e.g., weak, inept) and memory (e.g., dementia, forgetting) stereotypes. A control group saw similar stimuli without stereotype words. We controlled stereotype exposure, level of negativity, number of words, and stimulus size. Two-thirds of the stereotype group later reported awareness of the stimulus characteristics ("aware" group). Multiple measures of beliefs and memory were examined before and after stimulus exposure. The control and stereotype groups were comparable on beliefs and memory before working with the familiar materials. After exposure, 1) the stereotype group had higher story recall scores, and 2) the aware group showed the lowest perceived memory threat and the highest general memory evaluation. These results show that those who noticed the stereotypes counteracted their influence. Focusing on how to train stereotype awareness and response would be useful in future research.

\section{MINDFUL AGING: THE IMPACT OF TRAIT MINDFULNESS ON AGING STEREOTYPES}

S. Mallya, V. Huang, A.J. Fiocco, Psychology, Ryerson

University, Toronto, Ontario, Canada

With a growing aging population, it is important to understand factors that encourage or discourage healthy 